\title{
Inheritance of the Anatomy-Morphological Structure of the Stalk by Interspecific Hybrids of the Glycine L.
}

\author{
Margarita Kozak \\ Department of Biology, pl. Shaumyana 1, Astrakhan State University, Astrakhan 414000, Russia
}

Received: May 28, 2014 / Accepted: September 24, 2014 / Published: September 30, 2014.

\begin{abstract}
The research focuses on the study of anatomical and morphological stalk structure of soya interspecific hybrids of the third generation $\left(\mathrm{F}_{3}\right)$ between (Glycine $\max (\mathrm{L}$.) Merr.) and G. soja Sieb. et Zucc. in comparison with parent plant species. The parent plant species and interspecific hybrids were sowed and grew under similar conditions. The similarity of the anatomic structure of stalks of cultivated plants and Glycine soja (wild soya) proves the hypothesis the studied species have the same origin. However, the obtained results show the considerable degree of phylogenetic dissociation between the studied soya species. Interspecific hybrids inherit from $G$. soja the ability to high intensive growth. The G. soja use in practical selective breeding is of great interest.
\end{abstract}

Key words: Plant breeding and genetics, plant morphology and structure, Glycine max, Glycine soja (wild soya), interspecific hybrid, anatomic and morphological stalk.

\section{Introduction}

There are various hypotheses about the origin of cultivated and wild-growing and their phylogenetic relations [1-17, 18-21]. The species of wild-growing, Glycine soja Sieb. et Zucc., are actively involved in the selective breeding by their crossings with cultivated soya. The data on the inheritance of specific features of anatomical and morphological structure of plants as a result of hybridization of cultivated (Glycine $\max (\mathrm{L}$.) Merr.) and wild-growing species are required. It is efficient to use wild growing Glycine soja Sieb. et Zucc. in the crossing as a donor of genes conditioning multi-flower trait, early ripeness, complex immunity, high-protein content, and other valuable features. Along with these useful futures, the hybrids persistently inherit the twining stalk that is characteristic for wild-growing Glycine soja. The domination of unstable and twining stalks that the hybrids have produces certain difficulties in the selective breeding. Therefore, the study of the

Corresponding author: Margarita Kozak, Ph.D., professor, research field: cytogenetic plants. E-mail: mkozak@yandex.ru. principles of the mechanical elements in the structure of hybrid stalks and the principles of lignification of different anatomic elements is highly urgent. This paper deals with the research of the inheritance of anatomy-morphological structure of a stalk in the period of fruit formation when the formation of main anatomic elements completed.

\section{Experiment}

Wild and cultivated species of soya have much in common in the plant structure. Leaves and flowers grow in the axils along main stalk and branches. The stalk of both soya species is very branchy. The branches are sent out by stalk only from the level of the first and second leaflets to the first flower trusses. Branches do not grow in the nodes of the upper level.

The author produced the interspecific hybrids by the crossing of the cultivated soya (sort Beltskaya 656) and the wild growing Glycine soja gathered in the Amur region (the Far East of Russia). The emasculated flowers of cultivated soya were pollinated by pollen of wild growing Glycine soja. The formation of stalk anatomic structure was studied 
at each stage of the plant ontogenesis but in this paper, the author presents the results of the comparative analysis of anatomic structure of the plants that grew up and are in the phase of fruit formation.

The sort "Beltskaja 636" belongs to the Slavonic subspecies of cultivated soya [2], has an upright and steady stalk of $0.8-1.5 \mathrm{~m}$ height and yellow large seeds. Wild growing Glycine soja is an annual and herbaceous plant with a twining stalk with a length of 1.0-2.5 $\mathrm{m}$. The leaves are trifoliolate and fall before the beans are ripe. Leaves of leaflets are narrower than the leaves of cultivated species. Flowers are small, violet, beans are small and numerous, seeds are opaque and black. Plants are well adaptive for the environment.

The hybrids of the third generation with a steady and upright bottom stalk part and a twining middle and top stalk parts were studied. There has not ever been any hybrid of the third and fourth generation with a completely upright stalk. The anatomic structure of the bottom, middle and top stalk parts were studied. Test material was fixed in $75 \%$ alcohols. Anatomic slices were made with a straight razor and treated with phloroglucine and hydrochloric acid.

The figures were made by using of the drawing device PA-1 and a microscope of $8 \times 15$ magnification. The width (depth of formation) of basic anatomical elements was measured by the ocular micrometer.

\section{Results and Discussion}

The interspecific hybrids of the first generation had an intermediate phenotype in comparison with the parent plant species. The bottom stalk part of the hybrids almost did not differ in thickness, hardiness and fasciation from the stalk of cultivated species. The rest part of the stalk from the lower side branches to the top was twining and unstable. In the second and third generations, the plants of the following forms appeared:

- Plants with a twining stalk that is characteristic of the wild growing species; the next generations of the plants of this group appeared similar to the parent forms.

- Plants with an upright bottom stalk part that is characteristic of cultivated species but having a twining top part.

- The plants of this group were taken as a research object. In the fourth generation, they segregate into the following phenotype groups:

(1) Plants with an upright bottom stalk part but having a twining middle and top parts.

(2) Plants with a twining stalk as wild growing plants have.

(3) Small group (single plants) with an upright stalk as cultivated plants have.

The plants of the third group are of great interest for the selective breeding as in the seventh and eighth generations and in the backcrossing with cultivated soya, they transformed into homozygous forms that combine the stalk type of cultivated soya with an extremely high crop capacity (up to 320 and more beans per a plant). By backcrossing, these features can be transferred to the cultivated soya species. Thus, the author presents the peculiarities of anatomic stalk structure for the biotype that can be a parent to forms with an upright stalk.

The anatomic stalk structure of the studied forms is presented in the Figs. 1-3 (a, b, c). The remarkable similarity of the anatomic stalk structures of cultivated soya species (Fig. 1 (a, b, c)) and wild growing Glycine soja (Fig. 2 (a, b, c)) proves the hypothesis that the studied species have the same origin.

The author found out that at the first stages of plants growth their vascular system of both cultivated and wild species includes vascular bundles of a collateral open type which are close to each other. In the process of stalk transition to the secondary anatomic structure, the continuous central cylinder of a cyclic type is gradually forming. Referring to Cumbie, B. and Esau K. [22], state that secondary growth is characteristic of herbaceous plants of the family Fabaceae Lindl. and almost always occurs. 
However, along with the similarity of anatomic stalk structure of the studied species there are essential differences appeared during the phylogenesis of each species.

\subsection{The Characteristic of An Anatomic Stalk Structure of Cultivated Soya}

The bottom stalk part of cultivated soya (Fig. 1a) is characterized by strong development of the main anatomic elements especially mechanical tissue.

The stalk surface is covered by the single-layered epidermis consisting of cells that are tightly linked to each other and covered with a cuticle. The numerous outgrowths of epidermis cells create the hair-like covering of the stalk surface. The primary layer of the stalk cortex includes several (6-10) layers of collenchyma cells, carrying out not only mechanical, but also assimilation functions. They contain a significant number of chloroplasts. These cells are 1.5-2.0 times as large as epidermis cells. Under this layer, there are several layers of parenchyma cells with thin walls. The inner cell layer of the primary stalk cortex layer is endoderm consisting of cells that are tightly linked to each other and contain a considerable amount of starch granules. No signs of lignification or suberinization of endoderm cells were found. Endoderm adjoins to the continuous ring of the primary phloem fibers being on the periphery of stalk phloem. The width of this ring is $620-600 \mu \mathrm{m}$ (5-7 cell layers). The cell membrane of the primary phloem fibers lignifies greatly. As a result, the inside space of a cell has the form of a narrow hole.

The secondary phloem cells are arranged into irregular rows, relatively incoherently. The areas of the vascular tissue alternate with the areas of pith rays. The intercellular spaces are characteristic of the phloem layer (Fig. 1a).

The xylem ring of the bottom stalk part of the cultivated soya is strongly developed. All of the xylem elements including cells of pith rays lignify greatly.

Thus, the xylem layer represents a continuous ring of ligneous elements that are a steady base of the

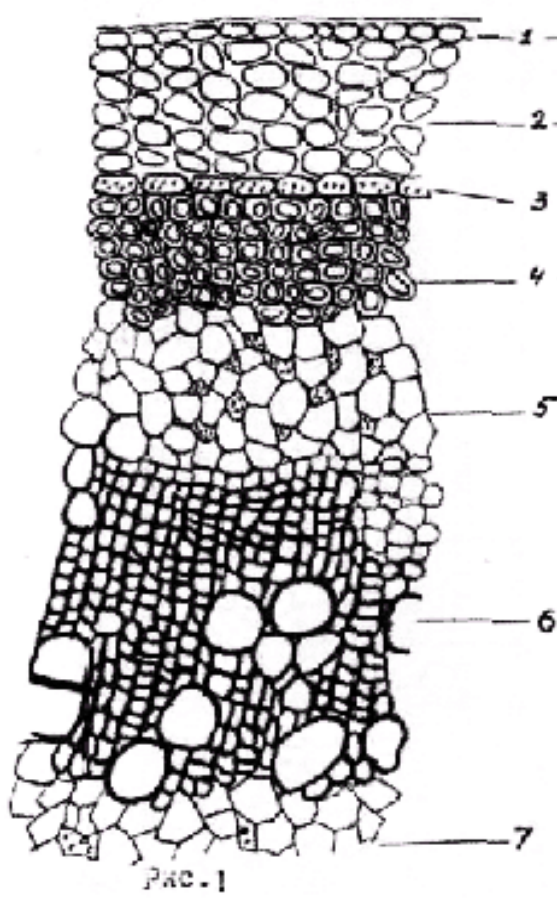

(a)

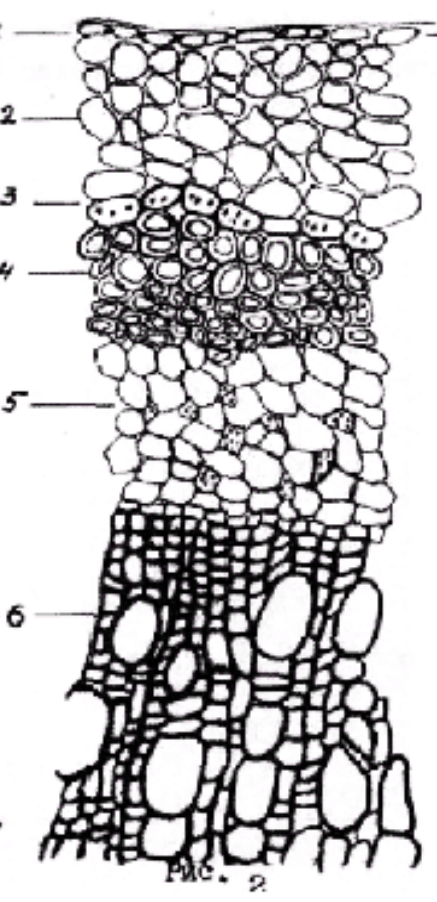

(b)

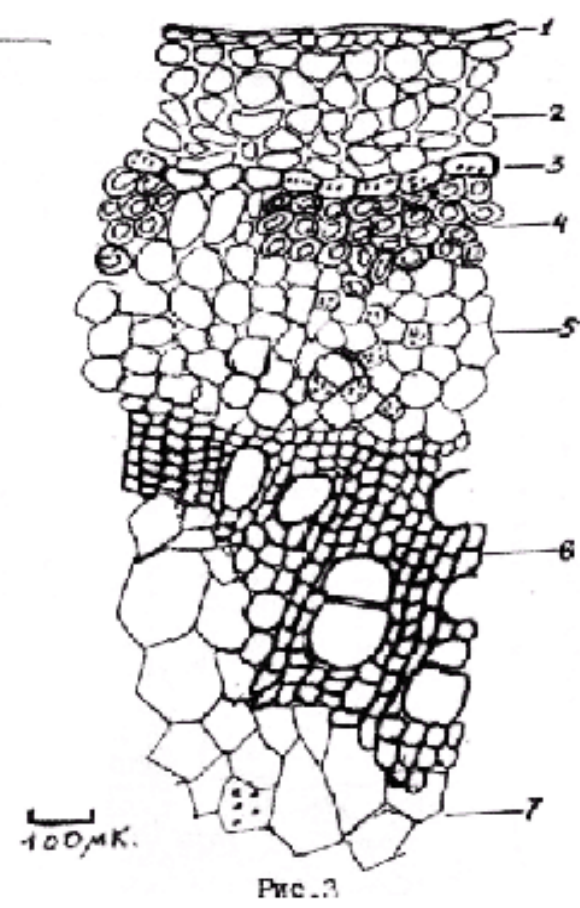

(c)

Fig. 1 (a, b, c) Anatomic structure of Glycine max (L.) Merr (Beltskaya 636) stalk in cross-section of bottom (a) middle (b) and top area (c): 1-epidermis, 2-primary cortex, 3-endoderm, 4-primary phloem fiber, 5-phloem, 6-xylem, 7-medulla. 
cultivated soya stalk. As a rule, the pith in the bottom stalk part dissolves and is replaced with the hollow space. Only the cells of peri-medullary zone remain unchanged. They remain to be living and fulfill the reserve functions. Thus, a powerful ring of ligneous xylem cells together with collenchyma and primary phloem fibers provide high hardiness of the cultivated soya stalk.

The middle part of the stalk (Fig. 1b) has little difference from the bottom part in the nature and depth of formation of main anatomic elements. Only the ring of xylem cells becomes much narrower (Table 1).

In the top part of the cultivated soya stalk (Fig. 1c), the cyclic nature of depth of main elements formation is expressed not clearly enough. The stalk retains its fascicular structure. The primary phloem fibers are arranged in a discontinuous ring with the width of 3-5 layers of cells, the walls of the fibers are lightly ligneous. The primary phloem fibers are located over the areas of the secondary phloem and xylem. The pith rays are very well-defined. Lignification of parenchyma of pith rays in the area of xylem is weak. The diameter of xylem vessels of the top stalk part is larger than in the bottom part.

The relatively weak development of the mechanical elements in the upper part of soya stalk causes a minor tendency to twining. The tendency to twining of the upper part of the stalk in some soya cultivars is stronger than in Beltchkaya 636. This feature is associated with dissection of fiber vascular bundles and absence of lignification of parenchyma cells of pith rays. This feature of the soya stalk anatomy shows the presence of wild type genes in genotype, most likely polymeric ones and confirms sufficiently close phylogenetic connections of representatives of these species.

In the study of soya stalk anatomic structure during the full fruit formation period (and later, at the end of the growing season), the author noticed low cell lignification of soft bast, especially in the upper and middle parts of stalk. This process is weaker at the bottom part of stalk. Other authors [23] have also marked the phloem lignification process while studying the anatomy of Helianthus L stalk. It is noted that phloem lignification can be observed at the end of vegetation and begins with the pericyclic fibers, at this time cambium disappears. This phenomenon has been noted in different plants by a number of researchers. G.I. Voroshilova observed lignification process of secondary phloem in soya stalk in full fruit formation [24]. K. Esau [22] also notes that cambium activity of stalk is reducing with phloem lignification.

\subsection{Anatomic Structure of Stalk Glycine soja (Wild Soya)}

Glycine soja stalk has almost the same anatomic elements (Fig. 2 (a, b, c)). The key difference from the cultivated soya stalk is a relatively weak development of mechanical elements and a lower degree of cell membrane lignification. Furthermore, a wild soya stalk has a thin cell layer of collenchyma in cortex (1-4 cell layers). However, the thin layer of chlorophyll-bearing parenchyma in cortex is significantly more developed.

The thickness of this layer is up to 11 cell rows and it borders collenchyma cells. Thus, cortex in the wild soya stalk is represented by the following elements: collenchyma (1-4 cell layers), a thin-walled chlorophyll-bearing parenchyma (3-11 cell layers) and endoderm, which is as typically pronounced as in cultivated soya.

Primary phloem fibers in the lower part of the stalk (Fig. 2a) form a broken ring (2-3 cell layers). The ring is continuous in the middle and upper parts of stalk but it has only one layer. Lignification degree of these cells is very low, is considerably weaker in every upper part.

Xylem ring in the lower part of the stalk is continuous but the lignification degree of parenchyma of pith rays is very low. Not all parenchyma of pith rays is exposed to lignification in xylem. Thickness of xylem and phloem layers in the lower part of stalk is 


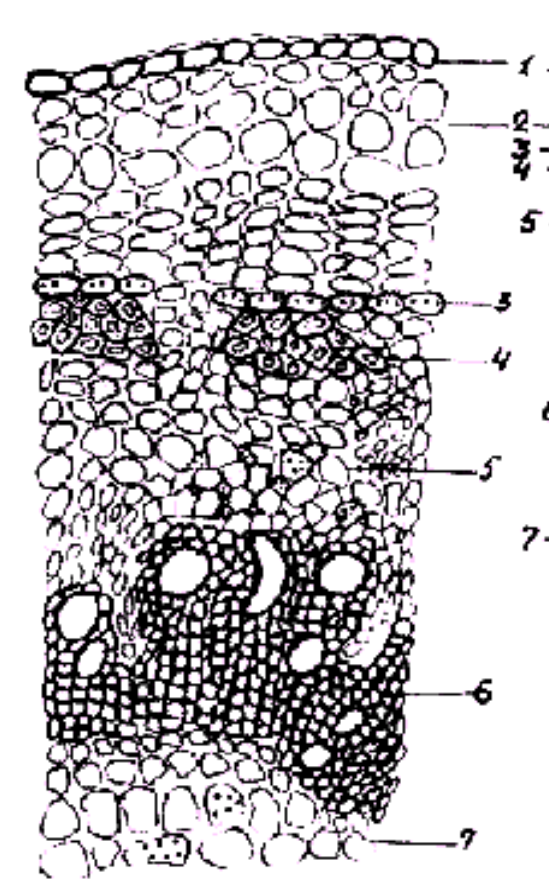

(a)

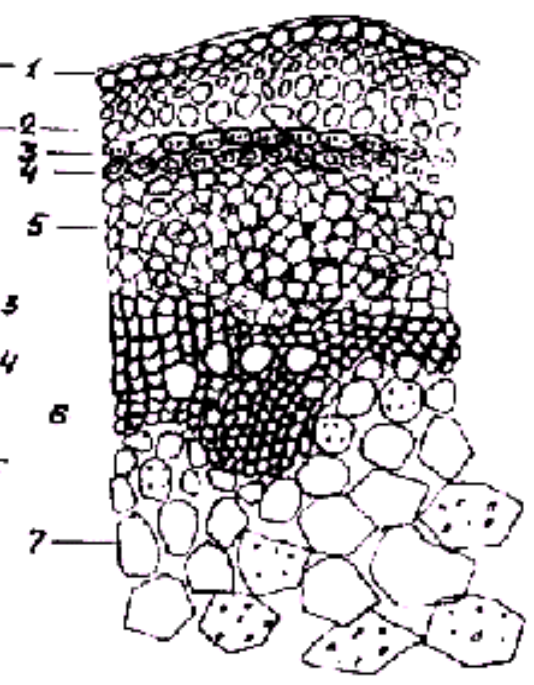

(b)

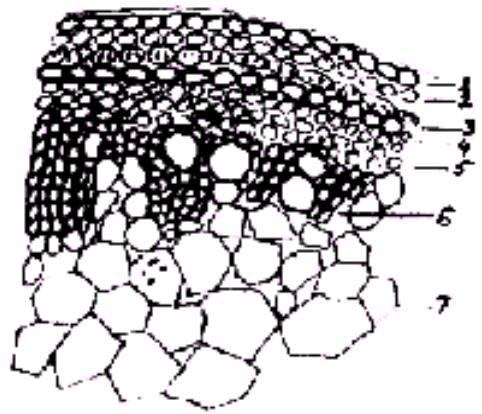

$\log _{10 \mathrm{x}}$.

Fig. 2 (a, b, c) Anatomic structure of Glycine soja (G. ussuriensis) stalk in cross-section of lower (a), middle (b) and top (c) parts: 1 - epidermis, 2—primary cortex, 3-endoderm, 4-primary phloem fiber, 5-phloem, 6—xylem, 7—medulla.

almost the same. Xylem dominates phloem in the middle and upper parts of the stalk (Fig. 2 (b, c)). There is no lignification of pith rays; the stalk retains its fasciculate structure. In general, the deposition thickness in the xylem of the wild soya half as large than cultivated soya has (Table 1). Glycine soja ( $G$. ussuriensis) has very low lignification of phloem.

\subsection{Anatomic Structure Characteristic of the Interspecific Hybrid Stalk}

The bottom part of the interspecific hybrid stalk is almost the same as the one of cultivated soya stalk (Fig. 3a). It is rough and upright. Middle and upper parts of stalk tend to twin, which is especially characteristic for the top part of the stalk. Anatomic structure of the stalk of the interspecific hybrid complies with its morphological features. It combines the structural features of wild and cultivated species (Fig. 3 (a, b, c)).

Anatomic structure of the stalk of hybrid forms at the bottom part (Fig. 3a) is almost the same as any kind of cultivated soya stalk; it has the same deposit thickness of basic anatomic elements and the degree of lignification. Table 1 shows comparative data on the development of basic anatomic elements in interspecific hybrid and original close species. Xylem ring is wide and continuous.

This layer of the hybrid is even wider than the one in cultivated soya. Xylem cell walls are considerably lignification. The xylem layer is twice thicker than the phloem layer. The structure of woody tissue of the middle and upper parts (Fig. 3 (b, c)) of the interspecific hybrid stalk is similar to Glycine soja. Ring structure of woody tissue is broken due to the fact that some parts of parenchyma of pith rays in xylem are not subject to lignification. Vessels of large diameter dominate in woody tissue. Degree of lignification of primary phloem fibers decreases regularly bottom-up. In the bottom part of the stalk it is as strong as in cultivated soya. Hybrid primary phloem fibers underlies in broken lots in the upper part of the stalk. The predominance of xylem elements and other mechanical elements over other elements of the anatomic structure provides stability of the hybrid 


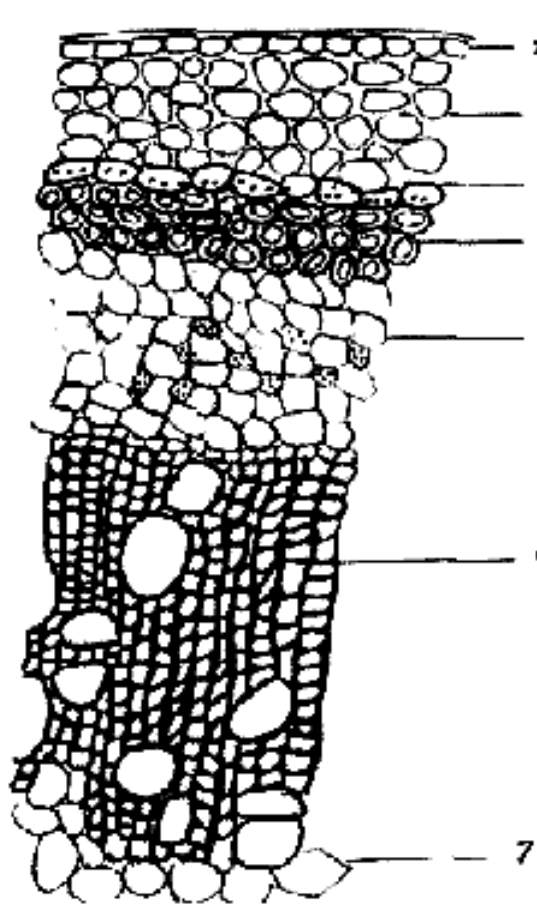

(a)

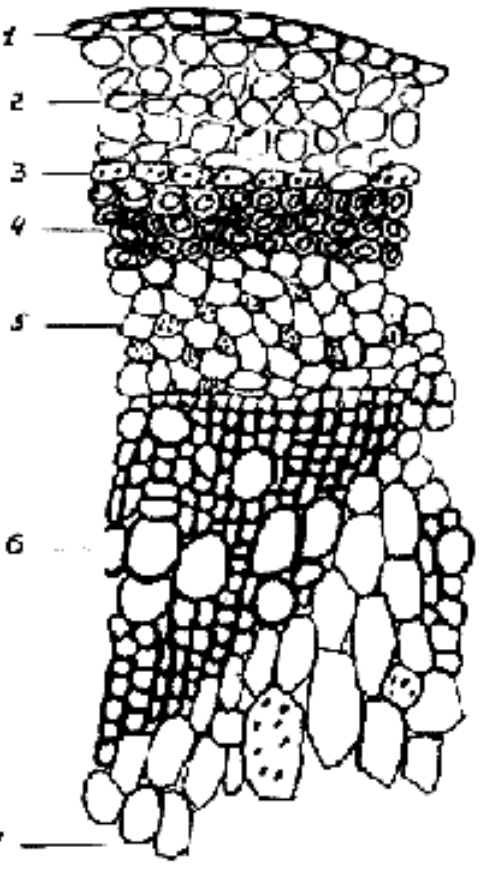

(b)

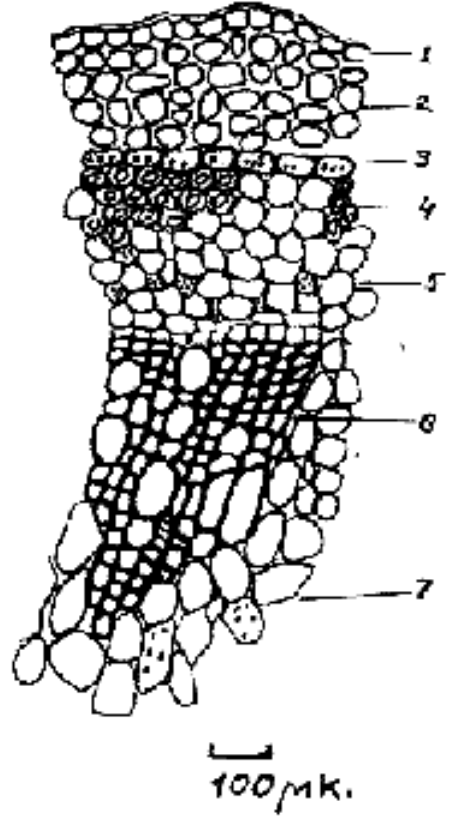

(c)

Fig. 3 (a, b, c) Anatomic structure of interspecific hybrid (Glycine max $\times$ G. soja) stalk in cross-section of lower (a), middle (b) and top (c) areas: 1-epidermis, 2-primary cortex, 3-endoderm, 4-primary phloem fiber, 5-phloem, 6-xylem, 7-medulla.

Table 1 Development of the basic anatomic elements in the structure of soya interspecific hybrid $G . \max \times G$. soja in comparison with original species

\begin{tabular}{|c|c|c|c|c|}
\hline \multirow{2}{*}{$\begin{array}{l}\text { Stalk area } \\
\text { (cross-section level) }\end{array}$} & \multicolumn{4}{|c|}{ Layer thickness (micrometer) } \\
\hline & Anatomic elements & $\begin{array}{l}\text { Interspecific hybrid } \\
\text { Glycine max } \times \text { G. soja }\end{array}$ & Glycine $\max$ & $\begin{array}{l}\text { Glycine soja } \\
\text { (G. ussuriensis) }\end{array}$ \\
\hline \multirow{5}{*}{$\begin{array}{l}\text { Bottom part of the } \\
\text { stalk }\end{array}$} & Collenchyma & 150 & 180 & $100-120$ \\
\hline & Parenchyma of the primary cortex & $70-100$ & $80-100$ & $300-350$ \\
\hline & Primary phloem fibers & 100 & $200-220$ & 100 \\
\hline & Phloem & 320 & 450 & 320 \\
\hline & Xylem & $750-800$ & $620-800$ & $350-450$ \\
\hline \multirow{5}{*}{$\begin{array}{l}\text { Middle part of the } \\
\text { stalk }\end{array}$} & Collenchyma & 150 & 200 & 20 \\
\hline & Parenchyma of the primary cortex & 100 & $100-200$ & 100 \\
\hline & Primary phloem fibers & 110 & $200-230$ & 20 \\
\hline & Phloem & 200 & 450 & 100 \\
\hline & Xylem (woody tissue) & $350-640$ & $750-780$ & $200-350$ \\
\hline \multirow{5}{*}{ Top area of the stalk } & Collenchyma & 250 & 50 & 0 \\
\hline & Parenchyma of the primary cortex & 150 & 50 & 50 \\
\hline & Primary phloem fibers & $70-100$ & $100-180$ & 20 \\
\hline & Phloem & 200 & 250 & 50 \\
\hline & Xylem (woody tissue) & $300-620$ & $400-800$ & $100-250$ \\
\hline
\end{tabular}

stalk bottom part. Formation of basic anatomic elements like the ones of the wild species and their low degree of lignification are responsible for twining of the upper part of the hybrid stalk. There is no cambium activity in the beginning of fruit formation period of the original species and the hybrid, however, the cambium is still preserved as a narrow strip of cells between the xylem and phloem. 


\section{Conclusions}

New data on the similarities and significant differences of the anatomic structure of the stalk of the studied species and interspecific hybrids prove not only the phylogenetic proximity but also a significant degree of evolutionary divergence. The deviation from the normal meiosis [5-8, 10] in the interspecific hybrids, reduced pollen fertility and significant size variations of pollen cell hybrids and that the author had noticed earlier, suggested lack of identity of genomes of the studied species Glycine max and Glycine soja and a significant degree of phylogenetic dissociation. Interspecific hybrids in the process of splitting can inherit the ability to high intensive growth and to produce large amounts of fruit (up to 320 or more beans per plant) from Glycine soja. The potential of using them as the original material for selective breeding is quite considerable.

\section{References}

[1] Fadeeva, T. S., and Burenin, V. I. 1990. Genetics of Cultural Plants: Leguminous, Vegetable, Cucurbitaceous. Leningrad: Agropromizdat 287.

[2] Enken, V. B. 1959. Soja. Russian: Sel'hozgiz. 622.

[3] Zhukovsky, P. M. 1971. Cultural Plants and Their Relatives. Leningrad: Kolos.

[4] Kozak, M. F. 1978. "Inheritance of Seed Color in Interspecific Soybean Hybrids." Genetics 14 (1): 36-43.

[5] Kozak, M. F. 1986. "Cytological Analysis of Meiosis in Microsporogenesis in Interspecific Soybean Hybrids." Cytology and Genetics 20 (3): 206-208.

[6] Kozak, M. F. 1990. "Results of Cytogenetic Researches of Hybrids of a Cultural and Wild-growing Soya." All-Union scientific research institute of plant growing of a name of N.I. Vavilov (VIR). Leningrad.135, Russian: 96-100.

[7] Kozak, M. F. 1993. "Mitotic Activity and Time Parameters of A Mitosis and Mitotic A Cycle at Two Kinds of A Soya and Interspecific Hybrids." Cytology and genetics 21 (1): 18-22.

[8] Kozak, M. F. 2004. Morphological Evolution and Cytogenetic Studies in Soybean. The monograph. Astrakhan: The Publishing house «Astrakhan University».

[9] Kozak, M. F. 2004. "The Mitotic Rhythms in Representatives of Soybean Glycine L." Cytology and Genetics 38 (6): 7-12.
[10] Kozak, Margarita. 2009. "Evolutionary Aspects Microsporogenesis and Microgametogenesis Interspecific Hybrids of Genus Glycine L." Soybean Genetics Newsletter 26 (4): 1-8.

[11] Kozak, Margarita. 2009. "Circadian Rhythm Mitosis Cells Apical Meristem A Root of Representatives of Genus Glycine L.” Presented at Conference VIII Beijing, China. The Chinese Academy of Agricultural Sciences Crop Science Society of China Organized by Institute of Crop Science, Chinese Academy of Agricultural Sciences.

[12] Kozak, Margarita. 2011. "Circadian Rhythm of Root's Apical Meristem Mitosis Cells of Soybean” Journal of Life Sciences (JLS) 5 (5): 364-368.

[13] Komarov, V. A. 1961. Origin of Cultural Plants. Leningrad: Publishing House of the USSR Academy of Sciences.

[14] Korsakov, N. I. 1972. Determinant of Kinds and Soya Versions. Leningrad: VIR.

[15] Sedova, T. S. 1985. "Wild-growing Relatives of A Soya an Initial Material for Selection." Nauch. That. bul. VIR of N.I. Vavilov 53: 17-19.

[16] Tihonchuk, P. V. 2004. Ecology-genetic Bases of Increase of Adaptive Potential of a Soya. Blagoveshchensk: The dissertation of the doctor of agricultural sciences (VAK 03.00.16).

[17] Shelko, G. 1995. Soya: the Genofond and Selection of Grain Befan Cultures. Sanct-Peterburg: VIR 196-322.

[18] Fucuda, Y. 1933. "Cytogenetically Studies on the Wild and Cultivated Manchurian Soybeans." Jap. J. Bot. 6 (5): 489-506.

[19] Hadley, H. H., and Hymowitz, T. 1973. "Speciation and Cytogenetic". In Soybeans: Improvement, Production and Uses, edited by Caldwell, B. E. Wisconsin: Madison 97-116.

[20] Herman, F. I. 1962. "A Revision of the Genus Glycine and Its Immediate Allies.” Dep. Agr. Techn. Bull 1268: 1-79.

[21] Singh, R. J., and Hymowitz, T. 1988. "The Genomic Relationship Between Glycine max (L.) Merr. and G. soja Sieb. and Zucc. as Revealed by Pachytene Chromosome Analysis." Theor. Appl. Genet. 76: 705-711.

[22] Esau, K. 1969. Anatomija of A Plant. Moscow: The World. 564.

[23] Aleksandrov, V. G., and Aleksandrova, O. G. 1931. 25 Years of Pedagogical and Public Work of Academician. Moscow: B.A. Keller. In Proceedings of the Academy of Sciences of the SSSN. Biological series: 88-103, Russian.

[24] Voroshilova, G. I. 1965. Formation of A Structure at Representatives of Two Kinds of A Soya in Them Ontogenesis. The author's abstract dissertations. 19, Russian. 